\title{
Effectiveness of Carbon Ion Radiation in Locally Advanced Pancreatic Cancer
}

\author{
Jakob Liermann ${ }^{1,2,3,4,5 *}$, Patrick Naumann ${ }^{1,2,3}$, Fabian Weykamp ${ }^{1,2,3}$, Philipp Hoegen ${ }^{1,2,3,4}$, \\ Juergen Debus ${ }^{1,2,3,4,5,6}$ and Klaus Herfarth ${ }^{1,2,3,4,5,6}$ \\ ${ }^{1}$ Department of Radiation Oncology, Heidelberg University Hospital, Heidelberg, Germany, ${ }^{2}$ Heidelberg Institute for Radiation \\ Oncology (HIRO), Heidelberg, Germany, ${ }^{3}$ National Center for Tumor Diseases (NCT), Heidelberg, Germany, ${ }^{4}$ Clinical \\ Cooperation Unit Radiation Oncology, German Cancer Research Center (DKFZ), Heidelberg, Germany, ${ }^{5}$ Heidelberg lon \\ Beam Therapy Center, Heidelberg, Germany, ${ }^{6}$ German Cancer Consortium (DKTK), partner site Heidelberg, German Cancer \\ Research Center (DKFZ), Heidelberg, Germany
}

Purpose: Effective treatment strategies for unresectable locally advanced pancreatic

OPEN ACCESS

Edited by: Giuseppe Magro, National Center of Oncological Hadrontherapy, Italy

Reviewed by:

Silvia Molinelli,

National Center of Oncological Hadrontherapy, Italy Amelia Barcellini, National Center of Oncological Hadrontherapy, Italy

*Correspondence: Jakob Liermann jakob.liermann@med.uniheidelberg.de

Specialty section: This article was submitted to

Radiation Oncology, a section of the journal

Frontiers in Oncology

Received: 12 May 2021 Accepted: 24 June 2021

Published: 14 July 2021

Citation: Liermann J, Naumann P, Weykamp F, Hoegen P, Debus J and Herfarth $K$ (2021) Effectiveness of Carbon Ion

Radiation in Locally Advanced Pancreatic Cancer.

Front. Oncol. 11:708884. doi: 10.3389/fonc.2021.708884 cancer (LAPC) patients are eagerly warranted. Recently, convincing oncological outcomes were demonstrated by carbon ion radiotherapy. Nevertheless, there is a lack of evidence for this modern radiation technique due to the limited number of carbon ion facilities worldwide. Here, we analyze feasibility and efficacy of carbon ion radiotherapy in the management of LAPC at Heidelberg lon Beam Therapy Center (HIT).

Methods: Between 2015 and 2020, 21 LAPC patients were irradiated with carbon ions with a total dose of $48 \mathrm{~Gy}$ (RBE) in single doses of 4 Gy (RBE). Three patients (14\%) were treated with concomitant chemotherapy with gemcitabine $300 \mathrm{mg} / \mathrm{m}^{2}$ body surface weekly. Toxicity rates were extracted from the charts. Overall survival, progression free survival, local control, and locoregional control were evaluated using Kaplan-Meier estimates.

Results: One patient developed ascites CTCAE grade III during radiotherapy, which was related to a later histologically confirmed metachronous peritoneal carcinomatosis. No further higher-graded toxicity could be observed. The most common symptoms were nausea and abdominal pain. After a median estimated follow-up time of 19.1 months, the median progression free survival was 3.7 months, and the median overall survival was 11.9 months. The estimated 1 -year local control and locoregional control rates were 89 and $84 \%$, respectively.

Conclusion: Carbon ion radiotherapy of LAPC patients is safely feasible. Local tumor control rates were high. Nevertheless, compared to historical data, an overall survival improvement could not be observed. This could be explained by the poor prognosis of the selected underlying patients that mostly did not respond to prior chemotherapy as well as the early and frequent emergence of distant metastases that demonstrate the necessity of additional chemotherapy in further studies.

Keywords: pancreatic cancer, carbon ion radiotherapy, particle therapy, locally advanced pancreatic cancer, irradiation, heavy ion 


\section{INTRODUCTION}

In pancreatic cancer, there is a lack of effective therapy options. Over the last decades, the limited five-year overall survival rate of $5-10 \%$ (1) could only be marginally challenged by modern treatment strategies. So far, surgical resection is the only curative therapy (2). However, in the majority of the patients, the tumor is deemed unresectable due to distant metastases or due to vessel involvement. Recently, Iacobuzio-Donahue et al. demonstrated local disease burden to be the cause of approximately one third of all pancreatic cancer related deaths (3). These findings demonstrate the urgent need for effective local treatment strategies.

In the last decades, several approaches tried to improve the oncological outcome of patients suffering from locally advanced pancreatic cancer (LAPC). One important goal in LAPC therapy is downstaging to gain secondary resectability. Because of the promising local effects of radiotherapy, multiple trials tested a potential benefit of chemoradiation over chemotherapy alone (4-6), but the data remain inconclusive. The Eastern Cooperative Oncology Group (ECOG) trial E4201 (6) and the Gastrointestinal Tumor Study Group (GITISG) trial 9283 (7) showed a slight beneficial effect of chemoradiation over chemotherapy. However, the LAP07 trial (4) revealed no difference in the median overall survival, although local tumor progression was significantly lower after chemoradiation compared to chemotherapy alone (32\%vs. $46 \%)$. The efficacy of chemotherapy could also be improved, recently. The most effective chemotherapy regime consisting of folinic acid, fluorouracil, irinotecan, and oxaliplatin (FOLFIRINOX) as part of LAPC therapy is correlated with a median overall survival of 24.2 months (8). However, many patients do not tolerate this aggressive chemotherapy regime due to comorbidity. As the results of chemoradiotherapy remain controversial, there is an ongoing discussion on the role of chemoradiation in the management of LAPC.

The observed limited effect of conventional radiotherapy in pancreatic cancer could partially be explained by the relatively low radiation doses applicable. This is due to the gastrointestinal tract which is highly sensitive to radiotherapy and which is situated adjacent to the pancreas $(9,10)$. To improve radiotherapy in LAPC, radiation doses should be increased. This could be reached by modern radiation techniques, such as stereotactic body radiotherapy, magnetic resonance (MR)guided radiotherapy or particle therapy (11-14). Using these techniques, higher doses can be applied to the tumor while respecting the dose limits for the adjacent organs at risk (OARs). Recently, Shinoto et al. presented convincing results of carbon ion radiotherapy in LAPC (15). In a dose-escalating trial, the observed median overall survival was 19.6 months after chemoradiation with 43.2-55.2 Gy (RBE) carbon ions applied in 12 fractions and combined with gemcitabine.

Carbon ion radiotherapy is known for two major advantages over conventional photon radiotherapy. First, there are physical characteristics making carbon ion radiotherapy highly conformal and precise. Energy deposition of particle therapy in irradiated tissue is different to the one of photon radiotherapy. Within a particle beam, there is a relatively low energy deposition in the entrance channel. The particles slow down and finally stop in a calculable depth, depending on their speed, represented by an increase of energy deposition and resulting in a maximum of energy deposition at a certain depth, the so-called Bragg Peak (16). There is almost no energy deposition behind this Bragg Peak. The resulting dose gradients in particle therapy are therefore very steep which makes the dose application highly precise. Second, carbon ions are known for a higher linear energy transfer (LET) and a higher relative biological effectiveness (RBE) compared to photons and even compared to protons (17). This is a biological advantage over photon radiotherapy as carbon ions are e.g. not as oxygen-dependent as photons. The biological characteristics of carbon ions could translate in improved therapy outcomes in so-far deemed radioresistant tumors (18).

In carbon ion radiotherapy planning, one of the most crucial aspects is the multifactorial dependency of the RBE resulting in different RBE values within the beam (19). Different carbon ion facilities use different RBE-models for carbon ion radiotherapy planning. Therefore, dose and therapy concepts cannot simply be transferred from one facility to another (20).

The advantages of carbon ion radiotherapy over conventional photon radiotherapy could improve oncological outcomes of LAPC patients as demonstrated by Shinoto et al. (15) In the present study, we analyze the feasibility and the efficacy of this promising radiation technique in the treatment of LAPC patients at Heidelberg Ion Beam Therapy Center (HIT).

\section{METHODS}

\section{Patients}

All patients presented with histologically confirmed pancreatic ductal adenocarcinoma or suspicious pancreatic tumor in imaging with correlating elevated tumor markers. To be included in the study, patients needed to suffer from inoperable LAPC without any sign of distant metastases (American Joint Committee on Cancer stage III). Two exceptions were made. One patient presented with a radiological suspicion of a distant lymph node metastasis, but the metastasis was not histologically confirmed at the time of radiotherapy. Another patient was included in the study, although he presented with hepatic metastases due to the fact that the hepatic metastases responded excellently to initial chemotherapy. A certain distance between the gastrointestinal tract and the tumor was not required. Any type and duration of prior chemotherapy was allowed. Recurrent cancer cases and patients participating in the ongoing PACK-trial (21) were excluded from the analysis. The inclusion criteria were chosen widely, as the institutional LAPC patient cohort treated with carbon ion radiotherapy is too small to define more specific subgroups.

\section{Target Volume Definition}

Planning imaging for radiotherapy was performed using fourdimensional native CT scans with a slice thickness of $3 \mathrm{~mm}$ to consider respiratory movement. Contrast-enhanced images were generated for differentiation of tumor and healthy tissue in 
delineation. Additionally, in six cases (29\%), fibroblast activation protein inhibitor-positron emission tomography (FAPI-PET) was performed prior to radiotherapy to improve target definition as recently demonstrated for locally recurrent pancreatic cancer (22). Contouring and radiotherapy planning were performed with the treatment planning system Syngo PT Planning (Siemens, Erlangen, Germany).

For delineation of the gross tumor volume (GTV), all applicable imaging was used to define the macroscopic tumor. Assumed microscopic tumor invasion was defined as clinical target volume (CTV). Therefore, the GTV was isometrically expanded by $6 \mathrm{~mm}$ and corrected considering anatomic boundaries such as non-infiltrated bone. Locoregional lymph nodes and the neuro-plexus were only part of the CTV when infiltrated. Considering respiratory movement, an internal target volume (ITV) was generated. The planning target volume (PTV) consisted of the ITV, enlarged by $5 \mathrm{~mm}$ in all directions $(7 \mathrm{~mm}$ in beam direction).

\section{Dose Prescription and Dose Constraints}

Patients were irradiated with a total dose of $48 \mathrm{~Gy}$ (RBE) applied in 12 fractions. The corresponding equivalent dose at $2 \mathrm{~Gy}$ (EQD2) is 61.7 Gy, assuming an $\alpha / \beta$-ratio of $5 \mathrm{~Gy}$. A dose maximum in the upper gastrointestinal tract of $43.2 \mathrm{~Gy}$ (RBE) should be respected. Underdosage of the planning target volume (PTV) to respect gastrointestinal constraints in challenging cases was decided upon individually case by case. A representative underdosage within the target volume is demonstrated in Figure 1. The dosage of the spinal cord was restricted to a maximum of $36 \mathrm{~Gy}(\mathrm{RBE})$, and the kidney volume receiving more than $24 \mathrm{~Gy}$ (RBE) was not allowed to exceed $20 \%$ of the whole organ.

\section{Treatment Delivery}

Carbon ion radiotherapy was applied with an intensitycontrolled raster scanning system for beam application at a rotating gantry. The first six patients $(29 \%)$ were situated in prone position to avoid a beam entry through the couch. After commissioning irradiation through the treatment table, the remaining 15 patients $(71 \%)$ could be treated in supine position. Accordingly, patients were predominantly situated on their back with the arms over the head (in Wing-Step mount) and with support in the back of the knee (knee cushion or ProStep mount). The laser-marked reference spots were defined by three small ink marks (Beekley spots). In all but one cases, two dorsal oblique radiation beams were used. Four-dimensional CT data of each patient were analyzed to evaluate the respiratory movement. In cases with large cranio-caudal target movement, gating was considered. In cases with an acceptable movement of the target, an ITV of all respiration phases was generated instead. In the presented patient cohort, finally, none of the patients was irradiated in a gating technique. Abdominal compression was not used to avoid a forced proximity of the gastrointestinal to the target volume. Image-guidance was performed through daily orthogonal X-rays and regular CT scans (at least once per week). If available, operation clips or stents were used for alignment. Otherwise, the spine was used to match X-rays and planning CT images. To consider organ movement and anatomical changes, a planning risk volume of the gastrointestinal was generated based on the fourdimensional CT data and patients needed to fast for at least $3 \mathrm{~h}$ prior to irradiation. If necessary, a new plan was generated based on the performed control CT. In the presented analysis, this was the case in two patients.

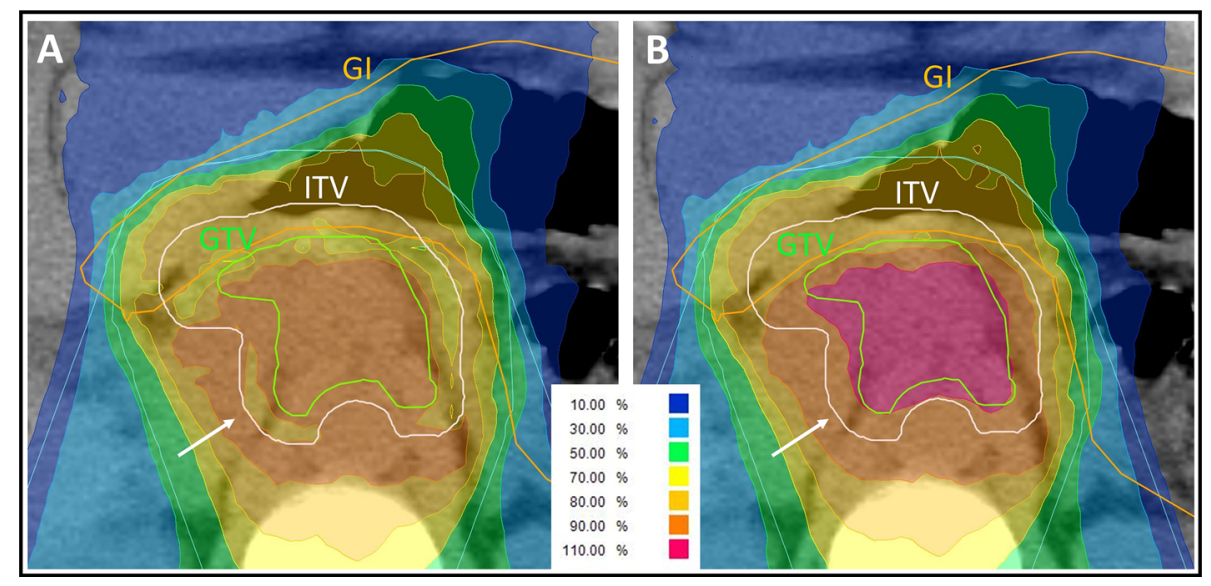

FIGURE 1 | Representative carbon ion radiation plan of a locally advanced pancreatic cancer patient using a clinical $\alpha / \beta$-ratio of 5 Gy for the internal target volume (ITV) and 2 Gy for the surrounding tissue in the treatment planning software's integrated relative biological effectiveness (RBE) model. The isodose lines are demonstrated in different colors. The percentages of the isodose lines shown in the legend correspond to the prescribed dose of 48 Gy (RBE) in 12 fractions. Underdosage of the ITV and of the gross tumor volume (GTV) needed to be accepted to respect the gastrointestinal (GI) constraints. (A) Due to the $\alpha / \beta$-ratio shift at the edge of the ITV, the peripheral ITV is irradiated with a lower biological dose than the surrounding tissue (white arrow), whereas the physically applied dose is increasing towards the center of the ITV. (B) Forward calculation using an $\alpha / \beta$-ratio of 2 Gy for all volumes in the RBE model. The dose distribution at the edge of the ITV is more homogenous (white arrow) but in this plan presentation, the GTV seems to be overdosed. This forward calculation helps in analyzing the plan but is not assumed to be correct, because of the missing biological assumption of the higher $\alpha / \beta$-ratio for the ITV. 


\section{Treatment Planning}

For biological plan optimization in carbon ion radiotherapy, different RBE values within the beam need to be considered. Therefore, the local effect model (LEM) I is integrated in the used treatment planning system (TPS). In LEM I, different $\alpha / \beta$-ratios for the ITV (first three patients: $\alpha / \beta=2 \mathrm{~Gy}$, remaining 18 patients: $\alpha / \beta=5 \mathrm{~Gy})$ and for the surrounding tissues $(\alpha / \beta=2$ Gy) were used.

The implementation of two different $\alpha / \beta$-ratios in the RBE model results in a complex dose distribution. In the ITV $(\alpha / \beta=5$ Gy), the physically applied dose needs to be higher than in the surrounding tissue $(\alpha / \beta=2 \mathrm{~Gy})$ to achieve the same biological dose. Thus, in the biological dose distribution prediction, there is a shift from the $\alpha / \beta$-ratio of 2 Gy (within the surrounding tissue) to the $\alpha / \beta$-ratio of $5 \mathrm{~Gy}$ (within the ITV). The biological dose in the peripheral margin of the ITV is deemed lower than the one of the surrounding tissue adjacent to the ITV edge (Figure 1A). This uncommon presentation of dose distribution seems inappropriate from the point of view of a conventional radiation oncologist. To facilitate plan evaluation, a forward calculation is performed using an $\alpha / \beta$-ratio of 2 Gy in LEM I for all structures including the ITV and the surrounding tissue (Figure 1B). As a consequence, the biological dose within the ITV is much higher in this forward calculation and should not be taken for granted. On the other hand, dose distribution seems more appropriate at the edge of the ITV. For adequate plan evaluation, both the actual plan and the forward calculation should be analyzed.

\section{Follow-up and Response Evaluation}

Follow-up was defined from the start of radiotherapy and was calculated using the reverse Kaplan-Meier method (23). Threemonthly contrast-enhanced CT scans and clinical visits were evaluated, whenever available. RECIST 1.1.-criteria were used in CT-based response evaluation. In-field tumor progression was denominated as "local tumor recurrence". "Regional tumor recurrence" was defined as out-field $(<90 \%$ of the prescribed dose) tumor progression of lymph nodes, soft tissue nearby or within the pancreas. "Distant tumor recurrence" was defined as any other tumor progression.

Overall survival (OS) was defined as time from the start of radiotherapy until death. Local control (LC) was defined from the start of radiotherapy until local tumor recurrence or last imaging available. Locoregional control (LRC) was defined from the start of radiotherapy until local or regional tumor recurrence or last imaging available. Progression free survival (PFS) was defined from the start of radiotherapy until any tumor progression or death or last imaging available.

\section{Toxicity}

Symptoms and complaints were graded according to the International Common Terminology Criteria for Adverse Events of the National Cancer Institute (NCI CTC AE), Version 5. Toxicity rates were extracted from the charts before the start of radiotherapy, during radiotherapy and at all available time points after the end of radiotherapy.

\section{Statistics}

OS, LC, LRC, and PFS were analyzed using the Kaplan-Meier method. Statistics and figures were performed with SPSS Statistics, version 27 (International Business Machines Corporation: IBM, New York, USA).

\section{Ethics}

The study was approved by the Ethics Committee of the University of Heidelberg, Germany (S-688/2020).

\section{RESULTS}

\section{Patient and Treatment Characteristics}

A total of 21 patients could be included in the analysis. Fourteen patients were treated with chemotherapy and one patient underwent partial tumor resection by whipple procedure prior to radiation. Prior chemotherapy regimen was heterogenous with a median of five cycles of FOLFIRINOX chemotherapy (range 1-10). Initial chemotherapy was predominantly performed as treatment of choice in LAPC patients or as neoadjuvant therapy to gain secondary operability. The majority of the patients presented in our institution due to missing tumor remission under chemotherapy or to avoid further chemotherapy. Radiotherapy with carbon ions was performed between January 2015 and July 2020. A total dose of 48 Gy (RBE) was delivered in 12 fractions in all cases. In three cases, concomitant chemotherapy with gemcitabine $300 \mathrm{mg} / \mathrm{m}^{2}$ body surface was administered weekly ( $2-3$ cycles). Patients that were known nonresponder to gemcitabine and patients that could not receive chemotherapy due to their general condition were not treated with concomitant chemotherapy. Additionally, the combination of gemcitabine chemotherapy and carbon ion radiotherapy was not tested to be safely applicable in 2015 which resulted in restrictive concomitant chemotherapy prescription at our institution. After radiotherapy, two initially inoperable patients were operated. One patient underwent total pancreatectomy (R1) after having developed local tumor recurrence after radiotherapy. In the second case, the tumor was deemed unresectable during operation and the procedure was completed without resection. During both operations, slight fibrosis was seen without leading to any interventional complications. Accordingly, the overall secondary resection rate was $5 \%$. In case of tumor progression during follow-up, patients were treated with different chemotherapy and immunotherapy regimen. Four patients were treated with gemcitabine and nabpaclitaxel, two patients with FOLFIRINOX, and further two patients with a combination of ipilimumab, nivolumab, and maraviroc. Detailed patient and treatment characteristics are summarized in Tables $\mathbf{1}$ and $\mathbf{2}$. A representative radiation plan is shown in Figure 2.

\section{Survival and Tumor Control}

After a median follow-up time of 19.1 months, the estimated 1year OS rate was $40.0 \%$ (Figure 3A). Two years after the start of radiotherapy, two of the observed patients were still alive and 
TABLE 1 | Patient characteristics.

\begin{tabular}{|c|c|c|}
\hline & $\mathbf{n}$ & $(\%)$ \\
\hline Number of patients & 21 & $(100)$ \\
\hline \multicolumn{3}{|l|}{ Sex } \\
\hline Male & 16 & (76) \\
\hline Female & 5 & (24) \\
\hline Age at radiotherapy (median in years, range) & $70(48-83)$ & \\
\hline \multicolumn{3}{|l|}{ Localization of initial pancreatic cancer } \\
\hline Pancreatic head & 13 & $(62)$ \\
\hline Pancreatic body & 7 & (33) \\
\hline Pancreatic tail & 1 & $(5)$ \\
\hline \multicolumn{3}{|l|}{ Initial AJCC* stage } \\
\hline IIB & 1 & (5) \\
\hline III & 17 & (81) \\
\hline IV & 3 & (14) \\
\hline \multicolumn{3}{|l|}{ Prior chemotherapy } \\
\hline FOLFIRINOX ${ }^{\circ}$ & 10 & $(48)$ \\
\hline FOLFIRINOX ${ }^{\circ}$, followed by gemcitabine + nab-paclitaxel & 4 & (19) \\
\hline None & 7 & (33) \\
\hline Time in months: prior chemotherapy (median, range) & $5(1-10)$ & \\
\hline \multicolumn{3}{|l|}{ Prior surgery } \\
\hline Whipple procedure (R2 resection) & 1 & $(5)$ \\
\hline None (apart from biopsy) & 20 & (95) \\
\hline \multicolumn{3}{|l|}{ Histology } \\
\hline Ductal adenocarcinoma & 18 & (86) \\
\hline unknown & 3 & (14) \\
\hline Secondary resection & 1 & (5) \\
\hline
\end{tabular}

${ }^{*}$ AJCC, American Joint Committee on Cancer.

${ }^{\circ}$ FOLFIRINOX, chemotherapy regimen consisting of folinic acid, fluorouracil, irinotecan, and oxaliplatin

TABLE 2 | Treatment characteristics.

\begin{tabular}{|c|c|c|}
\hline & $\mathbf{n}$ & $(\%)$ \\
\hline Radiotherapy & 21 & $(100)$ \\
\hline $\begin{array}{l}\text { Time in months: diagnosis to radiotherapy (median, } \\
\text { range) }\end{array}$ & $8(2-13)$ & \\
\hline \multicolumn{3}{|l|}{ Pre-radiotherapy AJCC* stage } \\
\hline III & 19 & (91) \\
\hline IV & 2 & (9) \\
\hline \multicolumn{3}{|l|}{ Radiation technique } \\
\hline Carbon ions, active raster-scanning & 21 & $(100)$ \\
\hline \multicolumn{3}{|l|}{ Prescribed dose } \\
\hline 48 Gy (RBE) in 12 fractions & 21 & $(100)$ \\
\hline \multicolumn{3}{|l|}{ Concurrent chemotherapy } \\
\hline Gemcitabine 300 mg/m² body surface weekly & 3 & $(14)$ \\
\hline None & 18 & (86) \\
\hline \multicolumn{3}{|l|}{ Patient position } \\
\hline Supine & 15 & $(71)$ \\
\hline Prone & 6 & (29) \\
\hline \multicolumn{3}{|l|}{ Volume in ccm (median, range) } \\
\hline GTV (Gross tumor volume) & $43.6(13.0-129.7)$ & \\
\hline CTV (Clinical target volume) & $128.4(26.1-323.3)$ & \\
\hline ITV (Internal target volume) & $183.4(48.3-583.5)$ & \\
\hline PTV (Planning target volume) & $303.2(96.7-812.0)$ & \\
\hline \multicolumn{3}{|l|}{ Number of radiation beams } \\
\hline 2 & 21 & $(100)$ \\
\hline \multicolumn{3}{|l|}{$\alpha / \beta$-ratio used in local effect model (LEM) I } \\
\hline 2 Gy & 3 & $(14)$ \\
\hline 5 Gy & 18 & (86) \\
\hline
\end{tabular}

${ }^{*}$ AJCC, American Joint Committee on Cancer. three patients were lost to follow-up. The observed median overall survival was 11.9 months. The corresponding 95\% confidence interval (CI) was 6.0-17.8 months. The estimated 1 -year PFS rate was $10 \%$ (Figure 3B), and the median PFS was 3.7 months (95\% CI 0.0-9.2).

Local progression could be observed in one patient 10 months after the start of radiotherapy (Figure 3C). The corresponding tumor could be resected but two months later distant metastases occurred. Regional and distant tumor recurrences were simultaneously observed in another patient that was treated with chemotherapy thereafter. No further locoregional tumor recurrence could be observed. One-year local control and oneyear locoregional control rates were 89 and $84 \%$, respectively.

\section{Toxicity}

During radiotherapy, there was an increase of reported nausea (13\% before radiotherapy vs. $48 \%$ during radiotherapy) and diarrhea. Nevertheless, these symptoms decreased after radiotherapy. One patient developed ascites CTCAE grade III during radiotherapy. Histopathological examination revealed underlying metachronous peritoneal carcinomatosis. No further higher-graded toxicity could be observed. After radiotherapy, $14 \%$ of the patients reported on fatigue. Toxicity rates are shown in Table 3.

\section{DISCUSSION}

To the best of our knowledge, we present the first European data on carbon ion radiotherapy in LAPC. The observed results demonstrate that carbon ion radiotherapy in pancreatic cancer is both feasible and well tolerable. Whereas convincing local tumor control rates could be achieved, OS rates were low due to a very short PFS of 3.7 months since distant metastases occurred early in most patients.

In all cases, radiation was completed as intended, and no radiation-induced higher graded toxicity was seen. Observed nausea and diarrhea could adequately be explained by radiation-induced mucositis of the gastrointestinal tract. Accordingly, these two symptoms decreased after the end of radiotherapy. Those findings are in line with the current literature. There are two retrospective analyses regarding carbon ion radiotherapy in LAPC $(24,25)$. Furthermore, Shinoto et al. published results of a prospective phase II doseescalating trial of 76 patients (15). In the mentioned studies, the most common toxicities included anorexia and gastrointestinal ulcer or bleeding. The latter could be observed in $<3 \%$ of the patients. In the present analysis, only one grade II bleeding of the lower gastrointestinal could be observed during follow-up. The patient was treated with anticoagulant therapy at the time of the event so that a correlation with the performed radiation seems to be less probable.

In the only prospective trial published so far, Shinoto et al. demonstrated a 1- and 2-year local tumor control rate of 92 and $83 \%$, based on CT imaging (15). We could confirm the local 

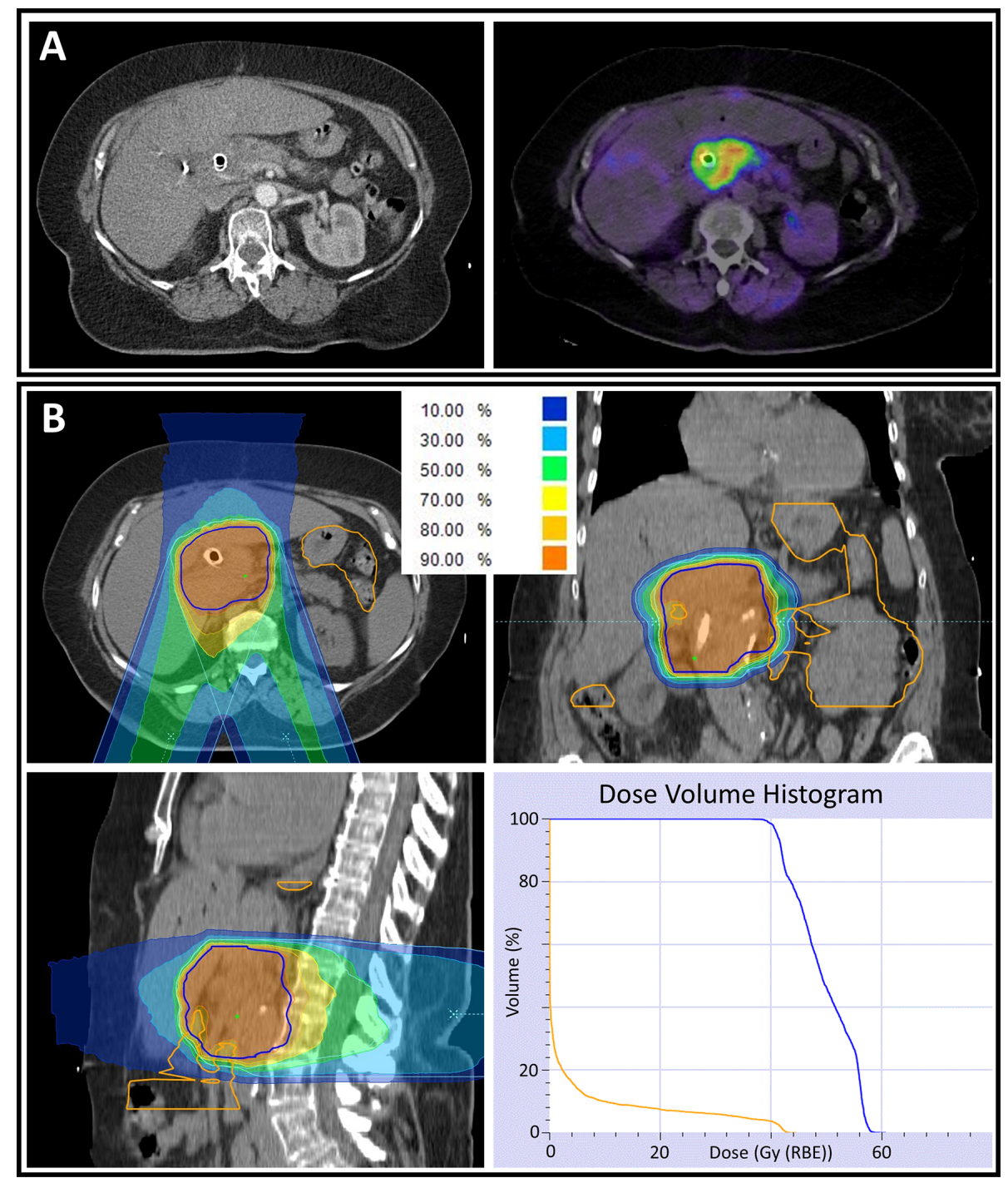

FIGURE 2 | (A) Computed tomography (CT) scan of a locally advanced pancreatic cancer patient demonstrating a pancreatic tumor mass with an implanted biliary metal stent (left). To improve target volume definition in radiation planning, a fibroblast activation protein inhibitor-positron emission tomography (FAPI-PET) with a high tumor to background contrast was performed and matched with CT data (right). (B) Radiation plan of the same patient in axial (upper left), coronal (upper right) and sagittal (lower left) CT slices. The percentages of the different-colored isodose lines correspond to the prescribed dose of 48 Gy (RBE) in 12 fractions. For all volumes, an $\alpha / \beta$-ratio of 2 Gy was used in the underlying relative biological effectiveness model. Partially, underdosage of the planning target volume (PTV, delineated in blue) needed to be accepted to respect the gastrointestinal (GI, delineated in orange) constraints. The dose volume histogram (lower right) demonstrates a 'stereotactic-radiotherapy-like' dose escalation within the PTV while respecting the GI constraints.

effectiveness of carbon ion radiotherapy with an observed 1-year local control rate of $87 \%$.

Nevertheless, OS results differed considerably. Shinoto et al. observed a median OS of 19.6 months (15). Kawashiro et al. presented an even higher median OS of 21.5 months after carbon ion radiotherapy in LAPC patients (25). This is almost twice as high as the observed 11.9 months of the present study. In the LAP 07 trial which compared photon radiotherapy-based chemoradiation with chemotherapy after induction therapy, the observed median overall was 12.8 months (4). Compared to these historical findings, we did not observe an OS improvement by carbon ion radiotherapy over photon radiotherapy in the present study.

One difference between the Japanese data and the present analysis is the underlying radiation dose concept. Kawashiro et al. irradiated with total doses of up to $55.2 \mathrm{~Gy}(\mathrm{RBE})$ delivered in 12 fractions. Furthermore, they described a correlation of higher-dosed radiotherapy and OS improvement (25). In the present analysis, we irradiated with a total dose of $48 \mathrm{~Gy}$ (RBE) in 12 fractions. However, dose concepts of different carbon ion facilities cannot be compared directly, which aggravates the interpretation of these findings. To be able to compare the 


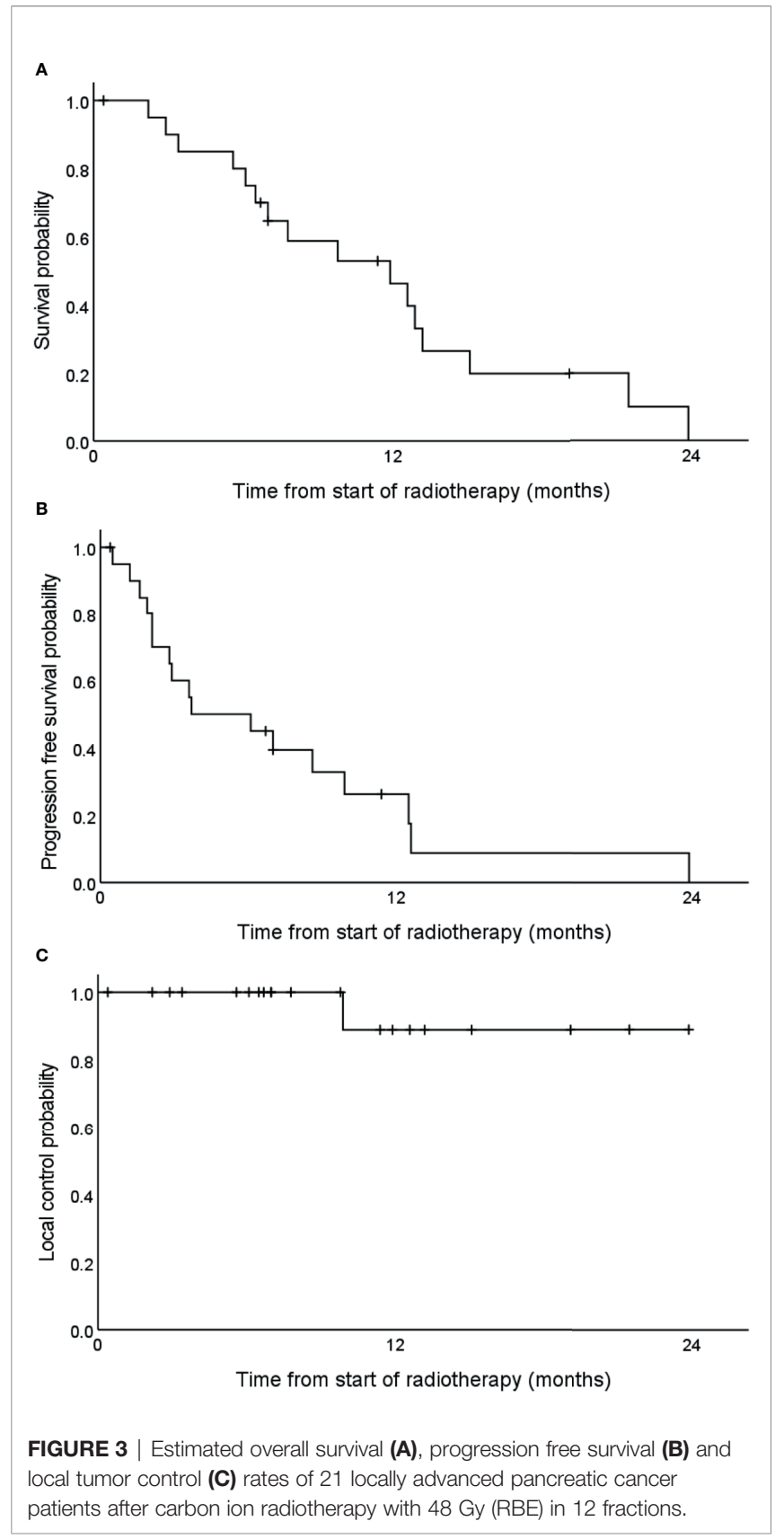

approach at the Heavy-Ion Medical Accelerator (HIMAC) at the National Institute of Radiological Science (NIRS) in Japan with the LEM I-based approach at our institution, Steinstrater et al. published conversion tables (20). According to these assumptions, the irradiated maximum single doses of $4.6 \mathrm{~Gy}$ (RBE) by Kawashiro et al. should correlate with single doses of 4.4-5.0 Gy (RBE) at our institution. Altogether, the radiation dose concept at our institution [single dose: $4.0 \mathrm{~Gy}(\mathrm{RBE})$ ] is supposed to be lower than the maximum one used in Japan. Nevertheless, considering the comparable local tumor progression rates of the different analyses, it seems rather unlikely that the diverse radiation dose concepts satisfactorily explain the OS differences.
TABLE 3 | Toxicity rates.

\begin{tabular}{|c|c|c|c|}
\hline Symptoms (NCI CTCAE grades) & $\begin{array}{c}\text { Before RT* } \\
\text { n (\%) }\end{array}$ & $\begin{array}{c}\text { During } \mathbf{R T}^{\star} \\
\text { n (\%) }\end{array}$ & $\begin{array}{c}\text { After } \mathrm{RT}^{\star} \\
\text { n (\%) }\end{array}$ \\
\hline \multicolumn{4}{|l|}{ Abdominal pain } \\
\hline 1 & $5(24)$ & $5(24)$ & $3(14)$ \\
\hline$\|$ & $5(24)$ & $4(19)$ & $2(9)$ \\
\hline \multicolumn{4}{|l|}{ Gastric hemorrhage } \\
\hline$\|$ & 0 & 0 & $1(5)$ \\
\hline \multicolumn{4}{|l|}{ Diarrhea } \\
\hline I & $2(9)$ & $3(14)$ & $1(8)$ \\
\hline \multicolumn{4}{|l|}{ Ascites } \\
\hline ॥ & 0 & 0 & $1(5)$ \\
\hline III & 0 & $1(5)$ & $1(5)$ \\
\hline \multicolumn{4}{|l|}{ Nausea } \\
\hline I & $2(9)$ & $6(29)$ & $1(5)$ \\
\hline$\|$ & $1(5)$ & $4(19)$ & $3(14)$ \\
\hline \multicolumn{4}{|l|}{ Dermatitis } \\
\hline I & 0 & $2(9)$ & 0 \\
\hline \multicolumn{4}{|l|}{ Fatigue } \\
\hline I & 0 & $2(9)$ & $1(5)$ \\
\hline$\|$ & 0 & 0 & $2(9)$ \\
\hline No complaints & $10(48)$ & $7(33)$ & $6(29)$ \\
\hline
\end{tabular}

${ }^{*} R T$, radiotherapy.

Shinoto et al. and Kawashiro et al. performed elective nodal irradiation and included the neuro-plexus region in the CTV (15, 25). At our institution, carbon ion radiotherapy volumes were kept as small as possible without elective nodal irradiation. The latter could lead to a higher rate of tumor recurrences. However, we did not observe high rates of locoregional tumor progression which partially contradicts this hypothesis.

Another significant difference between the Japanese trials and our study is the administration of chemotherapy. Shinoto et al. and Kawashiro et al. combined carbon ion radiotherapy with gemcitabine-based chemotherapy using doses of up to $1,000 \mathrm{mg} /$ $\mathrm{m}^{2}$. In the present study, only $14 \%$ of the patients were treated with simultaneous chemotherapy and administered gemcitabine doses were lower $\left(300 \mathrm{mg} / \mathrm{m}^{2}\right)$. The missing chemotherapy in the present treatment concept could explain the observed high rate of distant tumor progression and the relatively low OS rate.

Finally, there is a selection bias in the presented patient cohort. Since the final publication of the LAP 07 trial results, LAPC patients in Europe are predominantly treated with chemotherapy. Patients that are capable of being treated with intense chemotherapy regimen such as FOLFIRINOX will typically not be assigned to radiotherapy unless they did not respond to chemotherapy pretreatment. Sixty-seven percent of the irradiated patients of the present analysis were pre-treated with intense chemotherapy regimen and did not or did only poorly respond to pre-treatment. The median time from initial diagnosis to irradiation was 8 months. Altogether, the irradiated patient cohort consisted mostly of non-responding patients to pre-treatment with chemotherapy.

This selection bias could possibly explain the observed limited OS of the present study. The low secondary resection rate of 5\% supports this hypothesis. In a large meta-analysis of LAPC patients being treated with different modalities, the secondary resection rate was approximately 25\% (8). Furthermore, the median gross tumor size of $43.6 \mathrm{ccm}$ in the present analysis is 
almost three times larger than the observed tumor size of the prospective trial of Shinoto et al. $(14.8 \mathrm{ccm})(15)$ indicating a negative selection bias of the present patient cohort, too.

The present study has several limitations. First, the quality of data acquisition is limited because of the retrospective character of the analysis. Several patients did not regularly perform followup examinations, and no standardized quality of life questionnaires were used. Second, a sample size of 21 patients is small, which is due to the limited number of carbon ion facilities making carbon ion radiotherapy a rare treatment option. Third, the observed high local tumor control rate could be biased by the high rate of distant tumor progression during follow-up examination. It is possible that patients did not reach the criteria of local tumor progression because they deceased early after the end of radiotherapy.

On the other hand, a strength of the analysis is the reliability of the estimated overall survival due to the high number of reported deaths. Furthermore, the observed local tumor control rate and the toxicity rates seem to confirm the radiation dose concept as only one patient developed local tumor progression and no higher-graded radiation-induced toxicity was seen.

In conclusion, carbon ion radiotherapy in pancreatic cancer is well tolerable and locally effective. In the present analysis, an expected OS benefit over historical photon radiotherapy data could not be observed. This seems to be due to a negative selection bias of the described patient cohort. Considering the high rate of distant tumor progression, carbon ion radiotherapy should be combined with chemotherapy in future studies.

\section{REFERENCES}

1. Rawla P, Sunkara T, Gaduputi V. Epidemiology of Pancreatic Cancer: Global Trends, Etiology and Risk Factors. World J Oncol (2019) 10(1):10-27. doi: 10.14740 /wjon 1166

2. Doi R, Imamura $M$, Hosotani R, Imaizumi $T$, Hatori $\mathrm{T}$, Takasaki $\mathrm{K}$, et al. Surgery Versus Radiochemotherapy for Resectable Locally Invasive Pancreatic Cancer: Final Results of a Randomized Multi-Institutional Trial. Surg Today (2008) 38(11):1021-8. doi: 10.1007/s00595-007-3745-8

3. Iacobuzio-Donahue CA, Fu B, Yachida S, Luo M, Abe H, Henderson CM, et al. DPC4 Gene Status of the Primary Carcinoma Correlates With Patterns of Failure in Patients With Pancreatic Cancer. J Clin Oncol (2009) 27 (11):1806-13. doi: 10.1200/JCO.2008.17.7188

4. Hammel P, Huguet F, Laethem JL, Goldstein D, Glimelius B, Artru P, et al. Effect of Chemoradiotherapy vs Chemotherapy on Survival in Patients With Locally Advanced Pancreatic Cancer Controlled After 4 Months of Gemcitabine With or Without Erlotinib: the LAP07 Randomized Clinical Trial. JAMA (2016) 315(17):1844-53. doi: 10.1001/jama.2016.4324

5. Huguet F, Andre T, Hammel P, Artru P, Balosso J, Selle F, et al. Impact of Chemoradiotherapy After Disease Control With Chemotherapy in Locally Advanced Pancreatic Adenocarcinoma in GERCOR Phase II and III Studies. J Clin Oncol (2007) 25(3):326-31. doi: 10.1200/JCO.2006.07.5663

6. Loehrer PJSr., Feng Y, Cardenes H, Wagner L, Brell JM, Cella D, et al. Gemcitabine Alone Versus Gemcitabine Plus Radiotherapy in Patients With Locally Advanced Pancreatic Cancer: an Eastern Cooperative Oncology Group Trial. J Clin Oncol (2011) 29(31):4105-12. doi: 10.1200/JCO.2011.34.8904

7. Treatment of Locally Unresectable Carcinoma of the Pancreas: Comparison of Combined-Modality Therapy (Chemotherapy Plus Radiotherapy) to Chemotherapy Alone. Gastrointestinal Tumor Study Group. J Natl Cancer Inst (1988) 80(10):751-5. doi: 10.1093/jnci/80.10.751

8. Suker M, Beumer BR, Sadot E, Marthey L, Faris JE, Mellon EA, et al. FOLFIRINOX for Locally Advanced Pancreatic Cancer: a Systematic

\section{DATA AVAILABILITY STATEMENT}

The raw data supporting the conclusions of this article will be made available by the authors, without undue reservation.

\section{ETHICS STATEMENT}

The study was approved by the Ethics Committee of the University of Heidelberg, Germany (S-688/2020). Written informed consent for participation was not required for this study in accordance with the national legislation and the institutional requirements.

\section{AUTHOR CONTRIBUTIONS}

$\mathrm{JL}$ and $\mathrm{KH}$ designed and directed the project. JL gathered the data. JL analyzed the data and wrote the manuscript. PN, FW, $\mathrm{PH}, \mathrm{JD}$, and $\mathrm{KH}$ helped in finalizing the manuscript. JD and $\mathrm{KH}$ supervised the project. All authors contributed to the article and approved the submitted version.

\section{FUNDING}

JL is funded by the Physician-Scientist Program of Heidelberg University, Faculty of Medicine. We acknowledge financial support by the Open Access Publishing Fund of Heidelberg University.

Review and Patient-Level Meta-Analysis. Lancet Oncol (2016) 17(6):801-10. doi: 10.1016/S1470-2045(16)00172-8

9. Kavanagh BD, Pan CC, Dawson LA, Das SK, Li XA, Haken RKT, et al. Radiation Dose-Volume Effects in the Stomach and Small Bowel. Int J Radiat Oncol Biol Phys (2010) 76(3 Suppl):S101-107. doi: 10.1016/j.ijrobp.2009. 05.071

10. Marks LB, Yorke ED, Jackson A, Haken RKT, Constine LS, Eisbruch A, et al. Use of Normal Tissue Complication Probability Models in the Clinic. Int J Radiat Oncol Biol Phys (2010) 76(3 Suppl):S10-19. doi: 10.1016/ j.ijrobp.2009.07.1754

11. Koong AC, Christofferson E, Le QT, Goodman KA, Ho A, Kuo T, et al. Phase II Study to Assess the Efficacy of Conventionally Fractionated Radiotherapy Followed by a Stereotactic Radiosurgery Boost in Patients With Locally Advanced Pancreatic Cancer. Int J Radiat Oncol Biol Phys (2005) 63 (2):320-3. doi: 10.1016/j.ijrobp.2005.07.002

12. Liermann J, Shinoto M, Syed M, Debus J, Herfarth K, Naumann P. Carbon Ion Radiotherapy in Pancreatic Cancer: A Review of Clinical Data. Radiother Oncol (2020) 147:145-50. doi: 10.1016/j.radonc.2020.05.012

13. Rutenberg MS, Nichols RC. Proton Beam Radiotherapy for Pancreas Cancer. J Gastrointest Oncol (2020) 11(1):166-75. doi: 10.21037/jgo. 2019.03.02

14. van Sornsen de Koste JR, Palacios MA, Bruynzeel AME, Slotman BJ, Senan S, Lagerwaard FJ. MR-Guided Gated Stereotactic Radiation Therapy Delivery for Lung, Adrenal, and Pancreatic Tumors: a Geometric Analysis. Int J Radiat Oncol Biol Phys (2018) 102(4):858-66. doi: 10.1016/j.ijrobp.2018. 05.048

15. Shinoto M, Yamada S, Terashima K, Yasuda S, Shioyama Y, Honda H, et al. Carbon Ion Radiation Therapy With Concurrent Gemcitabine for Patients With Locally Advanced Pancreatic Cancer. Int J Radiat Oncol Biol Phys (2016) 95(1):498-504. doi: 10.1016/j.ijrobp.2015.12.362

16. Brown A, Suit H. The Centenary of the Discovery of the Bragg Peak. Radiother Oncol (2004) 73(3):265-8. doi: 10.1016/j.radonc.2004.09.008 
17. Suit H, DeLaney T, Goldberg S, Paganetti H, Clasie B, Gerweck L, et al. Proton vs Carbon Ion Beams in the Definitive Radiation Treatment of Cancer Patients. Radiother Oncol (2010) 95(1):3-22. doi: 10.1016/j.radonc.2010. 01.015

18. Schlaff CD, Krauze A, Belard A, O'Connell JJ, Camphausen KA. Bringing the Heavy: Carbon Ion Therapy in the Radiobiological and Clinical Context. Radiat Oncol (2014) 9(1):88. doi: 10.1186/1748-717X-9-88

19. Karger CP, Peschke P. RBE and Related Modeling in Carbon-Ion Therapy. Phys Med Biol (2017) 63(1):01TR02. doi: 10.1088/1361-6560/aa9102

20. Steinstrater O, Grun R, Scholz U, Friedrich T, Durante M, Scholz M. Mapping of RBE-Weighted Doses Between HIMAC- and LEM-Based Treatment Planning Systems for Carbon Ion Therapy. Int J Radiat Oncol Biol Phys (2012) 84(3):854-60. doi: 10.1016/j.ijrobp.2012.01.038

21. Liermann J, Naumann P, Hommertgen A, Pohl M, Kieser M, Debus J, et al. Carbon Ion Radiotherapy as Definitive Treatment in non-Metastasized Pancreatic Cancer: Study Protocol of the Prospective Phase II PACK-Study. BMC Cancer (2020) 20(1):947. doi: 10.1186/s12885-020-07434-8

22. Liermann J, Syed M, Ben-Josef E, Schubert K, Schlampp I, Sprengel SD, et al. Impact of FAPI-PET/CT on Target Volume Definition in Radiation Therapy of Locally Recurrent Pancreatic Cancer. Cancers (Basel) (2021) 13(4):796. doi: 10.3390/cancers 13040796

23. Shuster JJ. Median Follow-Up in Clinical Trials. J Clin Oncol (1991) 9(1):1912. doi: 10.1200/JCO.1991.9.1.191

24. Shinoto M, Terashima K, Suefuji H, Matsunobu A, Toyama S, Fukunishi K, et al. A Single Institutional Experience of Combined Carbon-Ion Radiotherapy and Chemotherapy for Unresectable Locally Advanced Pancreatic Cancer. Radiother Oncol (2018) 129(2):333-9. doi: 10.1016/ j.radonc.2018.08.026

25. Kawashiro S, Yamada S, Okamoto M, Ohno T, Nakano T, Shinoto M, et al. Multi-Institutional Study of Carbon-Ion Radiotherapy for Locally Advanced
Pancreatic Cancer: Japan Carbon-Ion Radiation Oncology Study Group (J-CROS) Study 1403 Pancreas. Int J Radiat Oncol Biol Phys (2018) 101 (5):1212-21. doi: 10.1016/j.ijrobp.2018.04.057

Conflict of Interest: JD received grants from Viewray Incorporated, The Clinical Research Institute GmbH (CRI), Accuray International Sarl, RaySearch Laboratories AB, Vision RT Limited, Merck Seono GmbH, Astellas Pharma $\mathrm{GmbH}$, Astra Zeneca GmbH, Siemens Healthcare GmbH, Solution Akademie GmbH, Egomed PLC Surrey Research Park, Quintiles GmbH, Pharmaceutical Research Associates GmbH, Boehringer Ingelheim Pharma GmbH\&CoKG, PTWFreiburg Dr. Pychlau GmbH, Nanobiotix SA, Accuray Incorporated, Bristol-Myer Squibb GmbH\&CoKG aA and Merck KHG aA. As chairman of HIRO (Heidelberg Institute of Radiation Oncology, Heidelberg, Germany) and a managing director of the NCT (National Center for Tumor Diseases) Heidelberg, Germany, Juergen Debus is responsible for collaborations with a multitude of companies and institutions. Juergen Debus is CEO of the HIT Betriebs-GmbH and a member of the board of trustees of the Physikalisch-Technische Bundesanstalt (PTB). He attended advisory board meetings of MERCK KGaA (Darmstadt).

The remaining authors declare that the research was conducted in the absence of any commercial or financial relationships that could be construed as a potential conflict of interest.

Copyright (c) 2021 Liermann, Naumann, Weykamp, Hoegen, Debus and Herfarth. This is an open-access article distributed under the terms of the Creative Commons Attribution License (CC BY). The use, distribution or reproduction in other forums is permitted, provided the original author(s) and the copyright owner(s) are credited and that the original publication in this journal is cited, in accordance with accepted academic practice. No use, distribution or reproduction is permitted which does not comply with these terms. 